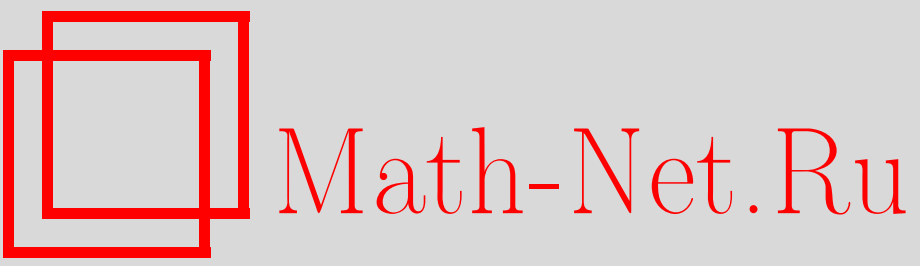

В. П. Петров, Г. С. Шаров, Классификация движений релятивистской струны с массивными концами, допускающих линеаризацию краевых условий, $Т M \Phi$, 1996, том 109, номер 2, 187-201

DOI: https://doi.org/10.4213/tmf1221

Использование Общероссийского математического портала Math-Net.Ru подразумевает, что вы прочитали и согласны с пользовательским соглашением

http: //www . mathnet.ru/rus/agreement

Параметры загрузки:

IP: 52.23 .180 .231

26 апреля 2023 г., 16:36:50 
ТЕОРЕТИЧЕСКАЯ

И МАТЕМАТИЧЕСКАЯ

ФИЗИКА

Том 109, № 2

ноябрь, 1996

\author{
В.П. Петров* , Г. С. Шаров*
}

\title{
КЛАССИФИКАЦИЯ ДВИЖЕНИЙ РЕЛЯТИВИСТСКОЙ СТРУНЫ С МАССИВНЫМИ КОНЦАМИ, ДОПУСКАЮШИХ ЛИНЕАРИЗАЦИЮ КРАЕВЫХ УСЛОВИЙ
}

\begin{abstract}
Классифицированы движения (мировые поверхности) релятивистской струны с массами на концах, допускающие параметризацию, в которой уравнения движения и краевые условия являются линейными вследствие пропорциональности параметра на траекториях концов струны натуральному параметру. Данные движения могут быть представлены в виде рядов Фурье по собственным функциям некоторого обобщения задачи Штурма-Лиувилля. Доказана полнота семейства этих собственных функций в классе $C$. Показано, что в пространствах Минковского размерностей $2+1$ и $3+1$ исследуемые движения сводятся к равномерному вращению прямолинейной струны или нескольких таких пространственно совмещенных струн (мировая поверхность - геликоид). В пространствах более высоких размерностей возможны иные нетривиальные движения рассматриваемого класса.
\end{abstract}

\section{ВВЕДЕНИЕ}

Теория струн сегодня представляет собой весьма обширную и разветвленную область математической физики $[1,2]$. В настояшей работе в центре внимания находится модель релятивистской струны с массивными концами $[1,3-5]$, которая была предложена для описания связанной сильным взаимодействием системы из двух кварков. Теоретический анализ движения такой струны сталкивается с рядом проблем. В частности, процедуру квантования $[1,2]$, разработанную для других струнных моделей и основанную на использовании ряда Фурье, здесь применять нельзя из-за сушественно нелинейных краевых условий, приводящих к, вообще говоря, непериодическому движению струны с массами на концах.

Одна из возможностей продвинуться в квантовании данной модели струны была реализована в работе [6], где рассматривались только те движения струны с массивными концами, которые допускают при определенном выборе координат на мировой поверхности линеаризацию краевых условий (при сохранении линейными уравнений движения). Это означает фактически введение координат, натурально параметризующих траектории концов струны.

K настояшему моменту окончательно не выяснено, насколько широк класс таких движений. Целью данной работы является точное определение его границ и внутренняя

* Тверской государственный университет 
классификация - исчерпьваюшее описание всех мировых поверхностей, допускающих упомянутую линеаризацию.

В первом разделе приведены основные уравнения, описывающие модель струны с массивными концами. Интересующий нас класс движений исследуется с помощью метода Фурье. Во втором разделе те же результаты получены и интерпретируются с помощью несколько иного подхода. В разделе 3 на основании строгих доказательств сделаны выводы о полноте семейства функций, используемого для разложения в ряд Фурье, а также классифицированы физические мировые поверхности с натурально параметризованными траекториями концов струны.

\section{1. УРАВНЕНИЯ ДВИЖЕНИЯ И КРАЕВЫЕ УСЛОВИЯ ДЛЯ РЕЛЯТИВИСТСКОЙ СТРУНЫ С МАССИВНЫМИ КОНЦАМИ. МЕТОД ФУРЬЕ}

Движение релятивистской струны с натяжением $\gamma$ и массами $m_{1}, m_{2}$ на конщах описывается действием $[1,3-6]$

$$
S=-\int_{\tau_{1}}^{\tau_{2}} d \tau\left\{\gamma \int_{\sigma_{1}(\tau)}^{\sigma_{2}(\tau)}\left[\left(\dot{X} X^{\prime}\right)^{2}-\dot{X}^{2} X^{\prime 2}\right]^{\frac{1}{2}} d \sigma+\sum_{a=1}^{2} m_{a} \sqrt{\left[\frac{d}{d \tau} X^{\mu}\left(\tau, \sigma_{a}(\tau)\right)\right]^{2}}\right\}
$$

Здесь $X^{\mu}(\tau, \sigma)$ - координаты точки струны в $D$-мерном пространстве Минковского $\mathbb{R}^{1, D-1} ; \sigma_{1}(\tau)$ и $\sigma_{2}(\tau)$ - внутренние координаты концов струны; $\dot{X}^{\mu}=\partial X^{\mu} / \partial \tau, X^{\prime \mu}=$ $\partial X^{\mu} / \partial \sigma ;(a b)=a^{\mu} b_{\mu}=\eta_{\mu \nu} a^{\mu} b^{\nu}-\left(\right.$ псевдо)скалярное произведение в $\mathbb{R}^{1, D-1} ;$ скорость света $c=1$.

Варьирование действия (1.1) дает уравнения движения струны и граничные условия, которые принимают простейший вид, если выбором координат $\tau$ и $\sigma$ сделать индуцированную метрику на мировой поверхности струны конформно-плоской, т.е. удовлетворяющей условиям ортонормальной калибровки

$$
\dot{X}^{2}+X^{\prime 2}=0, \quad\left(\dot{X} X^{\prime}\right)=0
$$

При выполнении этих условий уравнения движения становятся линейными:

$$
\ddot{X}^{\mu}-X^{\prime \prime \mu}=0
$$

а краевые условия на концах струны, нумеруемых индексом $a$, принимают вид

$$
\left.\left\{m_{a} \frac{d}{d \tau} \frac{\dot{X}^{\mu}+\sigma_{a}^{\prime}(\tau) X^{\prime \mu}}{\sqrt{\dot{X}^{2} \cdot\left(1-\sigma_{a}^{\prime 2}\right)}}+(-1)^{a} \gamma\left[X^{\prime \mu}+\sigma_{a}^{\prime}(\tau) \dot{X}^{\mu}\right]\right\}\right|_{\sigma=\sigma_{a}(\tau)}=0
$$

Зафиксируем внутренние уравнения траекторий концов струны в виде

$$
\sigma_{1}(\tau)=0, \quad \sigma_{2}(\tau)=\pi,
$$

что всегда можно сделать $[1,4]$, используя инвариантность выражений $(1.2)$ и $(1.3)$ относительно преобразований

$$
\tilde{\tau} \pm \tilde{\sigma}=f_{ \pm}(\tau \pm \sigma)
$$


Отметим, что условия (1.5) фиксируют свободу выбора функций $f_{ \pm}(\xi)$ не окончательно. Если $\phi(\xi)$ - произвольная $2 \pi$-периодическая гладкая функция с ограниченной производной, то замены переменных класса (1.6) с

$$
f_{+}(\xi)=f_{-}(\xi)=\xi+\phi(\xi), \quad \phi(\xi+2 \pi)=\phi(\xi), \quad\left|\phi^{\prime}(\xi)\right|<1
$$

сохраняют вид (1.5) уравнений коншов струны: $\tilde{\sigma}_{1}=0$ и $\tilde{\sigma}_{2}=\pi$. Произвол в выборе координат (1.6)-(1.7) на мировой поверхности можно интерпретировать как физическую несушественность "продольных колебаний" внутренних точек струны (в рамках действия (1.1) эти точки неразличимы).

Сосредоточим внимание на движениях рассматриваемой струны, допускающих параметризацию, которая, удовлетворяя условиям (1.2), обеспечивает на траекториях концов (1.5) пропорциональность параметра $\tau$ натуральному параметру $s=\int\left(\dot{X}^{2}\right)^{1 / 2} d \tau$ (собственному времени):

$$
\frac{d s}{d \tau}=\left.\sqrt{\dot{X}^{2}}\right|_{\sigma=\sigma_{a}}=C_{a}=\text { const }, \quad a=1,2 .
$$

Заметим, что к данному классу относятся движения с параметризацией, в которой $\chi_{1}(\tau)=\dot{X}^{2}(\tau, 0)-2 \pi$-периодическая функция, а $\chi_{2}(\tau)=\dot{X}^{2}(\tau, \pi)$ удовлетворяет условию $\chi_{2}(\tau)=$ const $\cdot \chi_{1}(\tau+\pi)$. Они сводятся к виду (1.8) с помошью замены (1.6)-(1.7).

Ограничение (1.8) приводит для исследуемого класса движений к линеаризации краевых условий (1.4):

$$
\left.\left(\ddot{X}^{\mu}-Q_{1} X^{\prime \mu}\right)\right|_{\sigma=0}=0,\left.\quad\left(\ddot{X}^{\mu}+Q_{2} X^{\prime \mu}\right)\right|_{\sigma=\pi}=0 .
$$

Здесь введены обозначения

$$
Q_{a}=C_{a} k_{a}=C_{a} \gamma / m_{a}, \quad a=1,2 ; \quad Q_{a}>0 .
$$

Константы $Q_{a}$ связаны с постоянными кривизнами траекторий $k_{a}=\gamma / m_{a}[5]$.

Множество решений уравнения (1.3), удовлетворяющих краевым условиям (1.9), ишем с помошью метода разделения переменных (метода Фурье) [6, 7], т.е. в виде линейной комбинации выражений $X^{\mu}(\tau, \sigma)=\alpha^{\mu} u(\sigma) T(\tau)$, где $\alpha^{\mu}$ - произвольный постоянный вектор. Подстановка этого выражения в (1.3) приводит к уравнениям $T^{\prime \prime}(\tau)+$ $\lambda T=0$ и

$$
u^{\prime \prime}(\sigma)+\lambda u=0 .
$$

При этом условия (1.9) преобразуются к виду

$$
\lambda u(0)+Q_{1} u^{\prime}(0)=0, \quad \lambda u(\pi)-Q_{2} u^{\prime}(\pi)=0 .
$$

Уравнение (1.11) с краевыми условиями (1.12) представляет некоторое обобщение задачи Штурма-Лиувилля, отличаюшееся от классической задачи $[7,8]$ наличием зависимости условий (1.12) от $\lambda$. 
Определим стандартным образом собственные функции $u_{n}(\tau)$ и собственные значения $\lambda_{n}$ задачи (1.11)-(1.12). Введем в $C_{[0, \pi]}$ скалярное произведение [6]

$$
\langle f, g\rangle \stackrel{\text { def }}{=} \int_{0}^{\pi} f(\sigma) \overline{g(\sigma)} d \sigma+\frac{f(0) \overline{g(0)}}{Q_{1}}+\frac{f(\pi) \overline{g(\pi)}}{Q_{2}},
$$

удовлетворяющее наряду с прочими обычными свойствами условию положительной определенности скалярного квадрата.

Покажем, что все собственные значения задачи (1.11)-(1.12) вешественны. Запишем с учетом (1.11) скалярный квадрат собственной функции:

$$
\lambda_{n}\left\langle u_{n}, u_{n}\right\rangle=-\int_{0}^{\pi} u_{n}^{\prime \prime}(\sigma) \overline{u_{n}(\sigma)} d \sigma+\lambda_{n} \frac{\left|u_{n}(0)\right|^{2}}{Q_{1}}+\lambda_{n} \frac{\left|u_{n}(\pi)\right|^{2}}{Q_{2}} .
$$

Выполняя дважды интегрирование по частям и реализуя условия (1.12), придем к равенству $\lambda_{n}\left\langle u_{n}, u_{n}\right\rangle=\overline{\lambda_{n}}\left\langle u_{n}, u_{n}\right\rangle$, что вследствие положительной определенности квадратичной формы $\langle u, u\rangle$ влечет $\lambda_{n}=\overline{\lambda_{n}}$. Очевидно, что все собственные функции данной задачи можно выбрать вещественными.

Убедившись с помошью непосредственной проверки, что отрицательных собственных значений задачи (1.11)-(1.12) не сушествует, введем для $\lambda_{n} \geq 0$ обозначение $\omega_{n}=$ $\sqrt{\lambda_{n}}$ и запишем соответствуюшие $\lambda_{n}$ нетривиальные решения (1.11)-(1.12) (собственные функции) $u_{n}$ в виде

$$
u_{n}(\sigma)=\cos \omega_{n} \sigma-\frac{\omega_{n}}{Q_{1}} \sin \omega_{n} \sigma, \quad n=0,1,2, \ldots, \quad \omega_{n}=\sqrt{\lambda_{n}} .
$$

Числа $\omega_{n}$ являются корнями трансцендентного уравнения, следующего из условий (1.12) $[6]$ :

$$
\left(Q_{1}+Q_{2}\right) \omega \cos \pi \omega=\left(\omega^{2}-Q_{1} Q_{2}\right) \sin \pi \omega .
$$

Каждому $\omega_{n}$ соответствует единственная с точностью до множителя функция $u_{n}$, а также две функции $T_{n}(\tau)=e^{ \pm i \omega_{n} \tau}, n \geq 1 ; T_{0}(\tau)=c_{1}+c_{2} \tau$.

Собственные функции (1.15) взаимно ортогональны в смысле скалярного произведения (1.13):

$$
\left\langle u_{n}, u_{p}\right\rangle=0, \quad n \neq p .
$$

Для доказательства достаточно записать $\lambda_{n}\left\langle u_{n}, u_{p}\right\rangle$ в виде, подобном (1.14), и, проделав те же операции, получить равенство $\lambda_{n}\left\langle u_{n}, u_{p}\right\rangle=\lambda_{p}\left\langle u_{n}, u_{p}\right\rangle$.

Из изложенного следует, что всякий сходящийся дважды дифференцируемьй ряд $\Phi$ уpьe

$$
X^{\mu}(\tau, \sigma)=\sum_{n=-\infty}^{\infty} \alpha_{n}^{\mu} u_{n}(\sigma) e^{-i \omega_{n} \tau}+p_{0}^{\mu} \tau, \quad \alpha_{-n}^{\mu}=\overline{\alpha_{n}^{\mu}}
$$

является решением задачи (1.3)-(1.9) (ограничимся вешественными $X^{\mu}$ ).

Рассматриваемый подход можно обобщить на предельные случаи нулевой и бесконечно большой массы $m_{a}$ на конце струны. При $m_{a}=0$ условия (1.9) и (1.12) приобретут соответственно вид $X^{\prime \mu}\left(\tau, \sigma_{a}\right)=0$ и $u^{\prime}\left(\sigma_{a}\right)=0$. Случай $m_{a} \rightarrow \infty$ будет рассмотрен ниже - он не сводится к равенству $Q_{a}=0$. 
Любопытно отметить, что задача (1.3)-(1.9) с $Q_{1}=0$ и $0<Q_{2}<\infty$ для каждой из координат $X^{\mu}$ полностью совпадает с описанной в [8] задачей о крутильных колебаниях упругого массивного цилиндрического стержня с закрепленным концом и с массивным диском на втором конце. При этом в роли $X^{\mu}(\tau, \sigma)$ выступает угол поворота стержня в сечении $\sigma$ в момент $\tau$. Случаю $Q_{1}>0$ соответствует подобный стержень с двумя дисками на концах.

Задача (1.3)-(1.9) для релятивистской струны отличается от ее механического аналога, в частности, тем, что ряд (1.18) имеет физический смысл только в том случае, когда его сумма удовлетворяет условиям ортонормальности (1.2), а также условиям (1.8). Чтобы лучше понять, насколько широк класс удовлетворяюших этим условиям мировых поверхностей, поставим предварительно вопрос о полном описании всех движений релятивистской струны. Например, для акустической струны с заданными краевыми условиями любое движение можно получить, задав начальное положение и начальную скорость ее точек $[7,8]$. Это утверждение справедливо и для струны с действием (1.1), т.е. для описания мировой поверхности достаточно задать на отрезке $[0, \pi]$ две функции $\rho^{\mu}(\sigma)$ и $v^{\mu}(\sigma)$, связанные с $X^{\mu}(\tau, \sigma)$ условиями

$$
X^{\mu}(0, \sigma)=\rho^{\mu}(\sigma), \quad \dot{X}^{\mu}(0, \sigma)=v^{\mu}(\sigma), \quad \sigma \in[0, \pi] .
$$

Условия (1.2) накладывают на функции $\rho^{\mu}(\sigma)$ и $v^{\mu}(\sigma)$ ограничение $\rho^{\prime 2}+v^{2}=0$, $\left(\rho^{\prime} v\right)=0$. Если оно выполнено, то мировая поверхность струны с начальными данными (1.19) и краевыми условиями (1.4) определяется однозначно [9]. Если же имеет место ряд (1.18), то коэффициенты $\alpha_{n}^{\mu}$ можно найти, подставив (1.18) в (1.19) и разложив $\rho^{\mu}(\sigma)$ и $v^{\mu}(\sigma)$ в ряды Фурье по функциям (1.15) с помошью скалярного произведения (1.13) с учетом (1.17):

$$
\alpha_{0}^{\mu}=\frac{\left\langle\rho^{\mu}, u_{0}\right\rangle}{\left\langle u_{0}, u_{0}\right\rangle}, \quad p_{0}^{\mu}=\frac{\left\langle v^{\mu}, u_{0}\right\rangle}{\left\langle u_{0}, u_{0}\right\rangle}, \quad \alpha_{n}^{\mu}=\frac{\left\langle\rho^{\mu}, u_{n}\right\rangle+i \omega_{n}^{-1}\left\langle v^{\mu}, u_{n}\right\rangle}{2\left\langle u_{n}, u_{n}\right\rangle}, \quad n \neq 0 .
$$

Следует отметить, что (в отличие от ситуации с акустической струной) заданной мировой поверхности релятивистской струны соответствует не единственная пара функций $\rho^{\mu}(\sigma)$ и $v^{\mu}(\sigma)$, а целое семейство. Это обусловлено произволом (1.6)-(1.7) в выборе параметров на мировой поверхности. Преобразование $\rho^{\mu}$ и $v^{\mu}$ при заменах (1.6)-(1.7) имеет довольно сложный вид, так как уравнения $\tau=0$ в старых и $\tilde{\tau}=0$ в новых координатах задают, вообше говоря, разные начальные кривые.

Имея в виду анализ этой проблемы и включение в рассмотрение случая $m_{a} \rightarrow \infty$ (на одном из конщов), прибегнем к подходу, изложенному в следуюшем разделе.

\section{2. РЯД ФУРЬЕ ДЛЯ ВЕКТОРА СКОРОСТИ КОНЦА СТРУНЫ}

Рассмотрим обшее решение уравнения (1.3)

$$
X^{\mu}(\tau, \sigma)=\frac{1}{2}\left[\Psi_{+}^{\mu}(\tau+\sigma)+\Psi_{-}^{\mu}(\tau-\sigma)\right] .
$$

Производные введенных здесь функций вследствие условий (1.2) изотропны:

$$
\Psi_{-}^{\prime 2}(\xi)=\Psi_{+}^{\prime 2}(\xi)=0
$$


Преобразуем краевые условия (1.4) (в соответствии с (1.5) $\sigma_{1}=0, \sigma_{2}=\pi$ ), подставив в них (2.1) с учетом (2.2):

$$
\frac{d}{d \tau} \frac{\Psi_{+}^{\prime \mu}\left(\tau+\sigma_{a}\right)+\Psi_{-}^{\prime \mu}\left(\tau-\sigma_{a}\right)}{\sqrt{2\left(\Psi_{+}^{\prime}\left(\tau+\sigma_{a}\right) \Psi_{-}^{\prime}\left(\tau-\sigma_{a}\right)\right)}}+\frac{(-1)^{a} \gamma}{2 m_{a}}\left[\Psi_{+}^{\prime \mu}\left(\tau+\sigma_{a}\right)-\Psi_{-}^{\prime \mu}\left(\tau-\sigma_{a}\right)\right]=0 .
$$

Первое из условий $(2.3)$ связывает $\Psi_{-}^{\prime \mu}(\xi)$ с $\Psi_{+}^{\prime \mu}(\xi)$, второе $-\Psi_{+}^{\prime \mu}(\xi+2 \pi)$ с $\Psi_{-}^{\prime \mu}(\xi)$. Эта связь весьма проста в предельных случаях $m_{a}=0$ и $m_{a} \rightarrow \infty$ [9]:

$$
\begin{aligned}
m_{a}=0 & \Rightarrow \Psi_{+}^{\prime \mu}\left(\xi+2 \sigma_{a}\right)=\Psi_{-}^{\prime \mu}(\xi) \\
m_{a} \rightarrow \infty & \Rightarrow \quad \Psi_{ \pm}^{\prime \mu}\left(\xi \pm 2 \sigma_{a}\right)=\left(2 W_{a}^{\mu} W_{a \nu}-\delta_{\nu}^{\mu}\right) \Psi_{\mp}^{\prime \nu}(\xi) .
\end{aligned}
$$

Здесь $\delta_{\nu}^{\mu}=\operatorname{diag}(1 ; 1 ; 1 ; \ldots), W_{a}^{\mu}=\mathrm{const}-$ единичный вектор скорости бесконечно тяжелого конца струны (такой конец движется прямолинейно с постоянной скоростью).

В случае конечных ненулевых масс удобно в качестве новых переменных ввести единичные векторы скорости концов струны:

$$
U_{a}^{\mu}(\tau)=\frac{\dot{X}^{\mu}\left(\tau, \sigma_{a}\right)}{\sqrt{\dot{X}^{2}\left(\tau, \sigma_{a}\right)}}=\frac{\Psi_{+}^{\prime \mu}\left(\tau+\sigma_{a}\right)+\Psi_{-}^{\prime \mu}\left(\tau-\sigma_{a}\right)}{\sqrt{2\left(\Psi_{+}^{\prime}\left(\tau+\sigma_{a}\right) \Psi_{-}^{\prime}\left(\tau-\sigma_{a}\right)\right)}}, \quad U_{a}^{2}(\tau)=1
$$

В работе [9] показано, что краевые условия (2.3) с учетом (2.6) можно свести к следуюшей системе уравнений $\left(k_{a}=\gamma / m_{a}\right)$ :

$$
\begin{aligned}
U_{1}^{\prime \mu}(\tau) & =k_{1}\left(\delta_{\nu}^{\mu}-U_{1}^{\mu} U_{1 \nu}\right) \Psi_{+}^{\prime \nu}(\tau) \\
U_{1}^{\mu}(\tau) & =W_{1}^{\mu}+\frac{k_{1}}{2}\left[\Psi_{+}^{\mu}(\tau)-\Psi_{-}^{\mu}(\tau)\right] \\
U_{2}^{\prime \mu}(\tau) & =k_{2}\left[\delta_{\nu}^{\mu}-U_{2}^{\mu}(\tau) U_{2 \nu}(\tau)\right] \Psi_{-}^{\prime \nu}(\tau-\pi), \\
U_{2}^{\mu}(\tau) & =\tilde{W}_{2}^{\mu}-\frac{k_{2}}{2}\left[\Psi_{+}^{\mu}(\tau+\pi)-\Psi_{-}^{\mu}(\tau-\pi)\right] .
\end{aligned}
$$

Интегрируя системы обыкновенных дифференциальных уравнений (2.7) и (2.9) и пользуясь соотношениями (2.8), (2.10) ( $W_{a}^{\mu}$ - постоянные интегрирования), мы можем продолжить на всю числовую ось функции $\Psi_{-}^{\mu}(\tau)$ и $\Psi_{+}^{\mu}(\tau)$, определенные на конечных отрезках с помощью начальных условий вида (1.19) [9].

Возводя в скалярный квадрат левую и правую части (2.7) или (2.9), учитывая при этом (2.2) и (2.6), получим соотношения

$$
\left(U_{a}(\tau) \Psi_{ \pm}^{\prime}\left(\tau \pm \sigma_{a}\right)\right)=k_{a}^{-1} \sqrt{-U_{a}^{\prime 2}(\tau)}
$$

Подставляя их в $(2.7),(2.9)$ и используя (2.8) и (2.10), мы придем к следующим выражениям, позволяющим определить $X^{\mu}(\tau, \sigma)$ по заданным $k_{a}$ и $U_{a}^{\mu}(\tau)[10]$ :

$$
\Psi_{ \pm}^{\prime \mu}\left(\tau \pm \sigma_{a}\right)=k_{a}^{-1}\left[\mp(-1)^{a} U_{a}^{\prime \mu}(\tau)+\sqrt{-U_{a}^{\prime 2}(\tau)} U_{a}^{\mu}(\tau)\right]
$$

Покажем, что формулы (2.7)-(2.12) при известных $m_{a}$ дают возможность восстановить с точностью до трансляций мировую поверхность струны, если вектор-функция 
$U_{1}^{\mu}(\tau)$ или $U_{2}^{\mu}(\tau)$ задана на отрезке с длиной $2 \pi$ (при вьполнении условий (1.5)). Пусть $0<m_{a}<\infty$ и функция $U_{1}^{\mu}(\tau)$ задана на отрезке [0,2 $2 \pi$. Найдя с помошью $(2.12)$ на том же отрезке $\Psi_{ \pm}^{\prime \mu}(\tau)$ и подставив $\Psi_{-}^{\prime \mu}$ в систему $(2.9)$, получим $U_{2}^{\mu}(\tau)$ на отрезке $[\pi, 3 \pi]$ (начальное условие для системы $U_{2}^{\mu}(\pi)=W_{2}^{\mu}$ для движений без разрывов $\dot{X}^{\mu}$ определяется выражением (2.6)). После этого с помошью соотношения (2.10) находим $\Psi_{+}^{\mu}(\tau)$ на отрезке $[2 \pi, 4 \pi]$ и, подставив $\Psi_{+}^{\mu}$ в $(2.7)$, продолжаем на этот отрезок $U_{1}^{\mu}(\tau)$. Процедура продолжения $U_{1}^{\mu}$ и $U_{2}^{\mu}$ может повторяться рекуррентно. В случаях $m_{a}=0$ и $m_{a} \rightarrow \infty$ использование (2.4) или (2.5) упрошает описанный механизм.

Итак, мы видим, что для полного описания движения струны достаточно задать одну из вектор-функций $U_{1}^{\mu}(\tau), U_{2}^{\mu}(\tau), \Psi_{+}^{\mu}(\tau)$ или $\Psi_{-}^{\mu}(\tau)$ на отрезке с длиной $2 \pi$. На этом отрезке допустима замена параметра $\tau$, приводяшая на мировой поверхности к репараметризации (1.6)-(1.7). Такой способ представления движения струны эквивалентен заданию функций $\rho^{\mu}(\sigma)$ и $v^{\mu}(\sigma)(1.19)$, но характеризуется более простой интерпретацией замен (1.6)-(1.7).

Обратимся теперь к тем движениям релятивистской струны, исследование которых является целью настояшей работы, т.е. к движениям с параметризацией, удовлетворяюшей условиям (1.8). Используя обозначения (2.1), (2.6) и выражение (2.11), эти условия можно переписать в ином виде:

$$
\sqrt{\dot{X}^{2}\left(\tau, \sigma_{a}\right)}=\sqrt{\left(\Psi_{+}^{\prime} \Psi_{-}^{\prime}\right) / 2}=\left(U_{a}(\tau) \Psi_{ \pm}^{\prime}\right)=k_{a}^{-1} \sqrt{-U_{a}^{\prime 2}(\tau)}=C_{a}
$$

(здесь $\left.\Psi_{ \pm}^{\prime}=\Psi_{ \pm}^{\prime}\left(\tau \pm \sigma_{a}\right)\right)$, или, учитывая (1.10):

$$
U_{1}^{\prime 2}(\tau)=-Q_{1}^{2}=\text { const }, \quad{U_{2}^{\prime}}^{2}(\tau)=-Q_{2}^{2}=\text { const } .
$$

Последние соотношения можно получить, используя определение кривизны $k_{a}$ как модуля вектора $d U_{a}^{\mu} / d s=U_{a}^{\prime \mu}(\tau) d \tau / d s$ и условие (1.8).

При выполнении эквивалентных (1.8) условий (2.13) или (2.14) уравнения (2.7), (2.9) и (2.12) приобретают линейный вид (здесь $( \pm)$ означает $(\tau \pm \pi)$, аргумент $(\tau)$ опушен):

$$
\begin{aligned}
U_{1}^{\prime \mu} & =k_{1} \Psi_{+}^{\prime \mu}-Q_{1} U_{1}^{\mu}, & \Psi_{ \pm}^{\prime \mu} & =k_{1}^{-1}\left[ \pm U_{1}^{\prime \mu}+Q_{1} U_{1}^{\mu}\right] ; \\
U_{2}^{\prime \mu} & =k_{2} \Psi_{-}^{\prime \mu}(-)-Q_{2} U_{2}^{\mu}, & \Psi_{ \pm}^{\prime \mu}( \pm) & =k_{2}^{-1}\left[\mp U_{2}^{\prime \mu}+Q_{2} U_{2}^{\mu}\right] .
\end{aligned}
$$

Будем искать решения этих уравнений, имеюшие на всей числовой оси вид гармоник. Зададим, например, вектор скорости 1-го конща $U_{1}^{\mu}(\tau)=\beta^{\mu} e^{-i \omega \tau}$. Из второго уравнения $(2.15)$ следует $\Psi_{ \pm}^{\prime \mu}(\tau)=\beta^{\mu} k_{1}^{-1}\left(Q_{1} \mp i \omega\right) e^{-i \omega \tau}$. Проинтегрировав это выражение и воспользовавшись $(2.10)$, найдем для $\omega \neq 0: U_{2}^{\mu}(\tau)=-\beta^{\mu} k_{1}^{-1} k_{2}[\cos \pi \omega+$ $\left.\left(Q_{1} / \omega\right) \sin \pi \omega\right] e^{-i \omega \tau}$. Подстановка $U_{2}^{\mu}(\tau)$ в любое из уравнений $(2.16)$ приводит к условию, которому должна удовлетворять частота $\omega$. Это условие, как и следовало ожидать, совпадает с (1.16).

Вследствие линейности уравнений (2.15)-(2.16) их решения - вектор-функции (2.6), удовлетворяюшие условиям (2.14), - можно искать в виде рядов

$$
U_{1}^{\mu}(\tau)=\sum_{n=-\infty}^{\infty} \beta_{n}^{\mu} e^{-i \omega_{n} \tau}, \quad U_{2}^{\mu}(\tau)=\sum_{n=-\infty}^{\infty} \Theta_{n} \beta_{n}^{\mu} e^{-i \omega_{n} \tau}, \quad \beta_{-n}^{\mu}=\overline{\beta_{n}^{\mu}}
$$

2 Теоретическая и математическая физика, т. 109, № 2, 1996 г. 
где

$$
\Theta_{n}=\left(C_{1} / C_{2}\right) u_{n}(\pi)=(-1)^{n}\left(k_{2} / k_{1}\right)\left[\left(\omega_{n}^{2}+Q_{1}^{2}\right) /\left(\omega_{n}^{2}+Q_{2}^{2}\right)\right]^{1 / 2}
$$

получены с помощью (1.16) и (1.10), $\omega_{n}$ - корни (1.16). Коэффициенты $\beta_{n}^{\mu}$ позволяют найти как $U_{1}^{\mu}$ и $U_{2}^{\mu}$, так и

$$
\Psi_{ \pm}^{\prime \mu}(\tau)=k_{1}^{-1} \sum_{-\infty}^{\infty} \beta_{n}^{\mu}\left(Q_{1} \mp i \omega_{n}\right) e^{-i \omega_{n} \tau}
$$

С другой стороны, дифференцируя ряд (1.18), мы получим

$$
\Psi_{ \pm}^{\prime \mu}(\tau \pm \sigma)=\dot{X}^{\mu} \pm X^{\prime \mu}=p_{0}^{\mu}-\sum_{n=-\infty}^{\infty} \alpha_{n}^{\mu} \omega_{n}\left(i \pm \frac{\omega_{n}}{Q_{1}}\right) e^{-i \omega_{n}(\tau \pm \sigma)}
$$

Сравнивая эти выражения, найдем взаимно однозначную с точностью до констант $\alpha_{0}^{\mu}$ и $C_{1}$ связь $\alpha_{n}^{\mu}$ с $\beta_{n}^{\mu}$ (то же следует из (1.18), (1.8) и (2.6)):

$$
p_{0}^{\mu}=C_{1} \beta_{0}^{\mu}, \quad \alpha_{n}^{\mu}=i C_{1} \omega_{n}^{-1} \beta_{n}^{\mu} \quad(n \neq 0), \quad C_{1}=Q_{1} / k_{1} .
$$

С помошью (2.19) можно найти $X^{\mu}(\tau, \sigma)$ по известному ряду $(2.17)$ для $U_{1}^{\mu}$.

Обратим внимание на особенности интересуюшего нас класса движений, характерные для случаев $m_{a}=0$ и $m_{a} \rightarrow \infty$. Например, при $m_{2} \rightarrow \infty$ вектор $U_{2}^{\mu}-$ постоянная величина: $U_{2}^{\mu}(\tau)=W_{2}^{\mu}$. Рассмотрим движения, удовлетворяюшие условиям (2.14), причем $Q_{2}=0$. Используя соотношения (2.15), а также (2.5) вместо теряющих смысл уравнений (2.16), мы придем к выводу, что единственная функция $U_{1}^{\mu}(\tau)$, определяюшая такое движение струны, подчиняется условию

$$
U_{1}^{\prime \mu}(\tau+2 \pi)+Q_{1} U_{1}^{\mu}(\tau+2 \pi)=A_{\nu}^{\mu}\left[-U_{1}^{\prime \nu}(\tau)+Q_{1} U_{1}^{\nu}(\tau)\right]
$$

где $A_{\nu}^{\mu}=2 W_{2}^{\mu} W_{2 \nu}-\delta_{\nu}^{\mu}$. Подстановка гармоник $U_{1}^{\mu}(\tau)=\beta^{\mu} e^{-i \omega \tau}$ в (2.20) приводит к соотношению

$$
A_{\nu}^{\mu} \beta^{\nu}=\frac{Q_{1}-i \omega}{Q_{1}+i \omega} e^{-2 \pi i \omega} \beta^{\mu} \equiv \Lambda(\omega) \beta^{\mu},
$$

которое означает, что $\beta^{\mu}$ должен быть собственным вектором матрицы $A_{\nu}^{\mu}$. Эта матрица имеет два собственных значения: $\Lambda=1$ с собственным вектором $W_{2}^{\mu}$ и частотами $\omega$, определяемыми уравнением $\Lambda(\omega)=1$, т.е.

$$
\omega / Q_{1}=-\operatorname{tg} \pi \omega
$$

и $\Lambda=-1$, которому соответствуют $D-1$ линейно независимых собственных векторов $\beta^{\mu}$, ортогональных $W_{2}^{\mu}$, с частотами, удовлетворяюшими уравнению

$$
\omega / Q_{1}=\operatorname{ctg} \pi \omega \quad\left(\left(W_{2} \beta\right)=0\right) .
$$

Обозначив через $\tilde{\omega}_{n}$ корни уравнения $(2.21)$ и через $\omega_{n}$ корни уравнения $(2.22)$, получим решение (2.20) в виде ряда

$$
U_{1}^{\mu}(\tau)=\sum_{n=-\infty}^{\infty}\left(c_{n} W_{2}^{\mu} e^{-i \tilde{\omega}_{n} \tau}+\beta_{n}^{\mu} e^{-i \omega_{n} \tau}\right), \quad\left(W_{2} \beta_{n}\right)=0 .
$$


Уравнение (2.22) совпадает с (1.16), если в последнем положить $Q_{2}=0$ (что соответствует $\left.m_{2} \rightarrow \infty\right)$. В то же время наличие уравнения $(2.21)$ и условия $\left(W_{2} \beta\right)=0$ кажется парадоксальным.

Объясняет ситуацию тот факт, что выполнение условия (2.14) $Q_{2}=0$ обусловлено равенством $k_{2}=\gamma / m_{2}=0$ и не требует выполнения для этого конца условия (1.8) (см. (1.10)). Следовательно, для движений (2.23) изначально не предполагалась пропорциональность параметра на траектории 2-го конца натуральному, поэтому для них нельзя считать справедливыми выражения (1.9) и (1.16) (имеет место "частичная" справедливость - не для всех $\beta^{\mu}$ ).

В противоположном предельном случае $m_{2}=0$, используя (2.4), можно получить уравнение, отличающееся от (2.20) заменой матрицы $A_{\nu}^{\mu}$ на единичную $\delta_{\nu}^{\mu}$. Для всех $\beta^{\mu}$ частоты гармоник определяются уравнением (2.21), которое может быть получено при использовании $X^{\prime \mu}(\tau, \pi)=0$ в качестве краевого условия (1.9) или просто предельным переходом $Q_{2} \rightarrow \infty$ в (1.16).

Как было сказано вьше, всякую мировую поверхность струны вида (1.1) можно определить, задав, например, единичную вектор-функцию $U_{1}^{\mu}(\tau)$ на отрезке $[0,2 \pi]$. С помощью замены параметра $\tau$, эквивалентной замене (1.6)-(1.7), всегда можно добиться на этом же отрезке вьполнения первого из условий (2.14). При этом оба условия (2.14) на всей числовой оси, вообше говоря, выполнены не будут. Другими словами, для произвольного движения струны в калибровке (1.2) можно ввести параметр, пропорциональный натуральному лишь на конечном отрезке траектории одного конца (с длиной $\Delta \tau=2 \pi$ при выполнении условий (1.5)).

Выясним, насколько ограничивает класс движений требование выполнения условий (2.14) или (1.8) на всей числовой оси.

\section{3. ПОЛНОТА СЕМЕЙСТВА СОБСТВЕННЫХ ФУНКЦИЙ И КЛАССИФИКАЦИЯ МИРОВЫХ ПОВЕРХНОСТЕЙ}

Покажем, что любое движение релятивистской струны (1.1), которое в параметризации с ортонормальной калибровкой (1.2) удовлетворяет условию (1.8), представимо в виде ряда Фурье (1.18), сходящегося к $X^{\mu}$. Для каждого такого движения условиями (1.19) определены функции $\rho^{\mu}(\sigma)$ и $v^{\mu}(\sigma)$ (для разных движений разные [9]), и с помошью (1.20) можно найти коэффишиенты ряда Фурье. Следовательно, необходимо доказать полноту семейства функций (1.15), например, в классе непрерывных функций.

Теорема 1. Система собственных функиий (1.15) краевой задачи (1.11)-(1.12) (где $\left.Q_{1}, Q_{2}>0\right)$ полна в классе $C_{[0, \pi]}$.

ДоКАЗАТЕЛЬСТво. Поставим в соответствие краевой задаче (1.11)-(1.12) “дуальную” задачу

$$
w^{\prime \prime}+\lambda w=0, \quad Q_{1} w(0)-w^{\prime}(0)=0, \quad Q_{2} w(\pi)+w^{\prime}(\pi)=0,
$$

которая является классической задачей Штурма-Лиувилля [7]. Собственные значения задач (1.11)-(1.12) и (3.1) $\lambda_{n}=\omega_{n}^{2}, n \geq 1$, совпадают (у последней отсутствует значение $\left.\lambda_{0}=0\right)$, частоты $\omega_{n}$ определяются одним и тем же уравнением (1.16). 
Собственные функции задачи (3.1) (ими могут служить производные функций (1.15)) выберем в виде

$$
w_{n}(\sigma)=\sin \omega_{n} \sigma+Q_{1}^{-1} \omega_{n} \cos \omega_{n} \sigma, \quad w_{n}=-\omega_{n}^{-1} u_{n}^{\prime}(\sigma), \quad n=1,2 \ldots
$$

Покажем, что всякую непрерывную функцию $f(\sigma)$ можно приблизить в норме $C$ функцией $\tilde{f}(\sigma)$ более высокого класса гладкости, удовлетворяющей определенным краевым условиям $-\forall \varepsilon>0, \forall f \in C_{[0, \pi]} \exists \tilde{f} \in C_{[0, \pi]}^{3}$ :

$$
Q_{1} \tilde{f}^{\prime}(0)-\tilde{f}^{\prime \prime}(0)=0, \quad Q_{2} \tilde{f}^{\prime}(\pi)+\tilde{f}^{\prime \prime}(\pi)=0, \quad\|f-\tilde{f}\|_{C}<\varepsilon / 2 .
$$

Для этого достаточно, используя теорему Вейерштрасса, приблизить $f$ многочленом $P(\sigma)$ так, что $\|f-P\|_{C}<\varepsilon / 4$, и положить $\tilde{f}=P(\sigma)+\Delta_{0}(\sigma)+\Delta_{\pi}(\sigma)$. Здесь $\Delta_{0}(\sigma)$ и $\Delta_{\pi}(\sigma)$ - малые поправки, обеспечиваюшие выполнение краевых условий $(3.3)$ (если $P(\sigma)$ им не удовлетворяет). Можно взять, например, $\Delta_{0}(\sigma)=\tilde{\varepsilon} e^{-A \sigma^{2} /(\pi-\sigma)}$, где $\tilde{\varepsilon}=$ $(\varepsilon / 4) \operatorname{sign} \Pi, A=2 \pi|\Pi| / \varepsilon, \Pi=P^{\prime \prime}(0)-Q_{1} P^{\prime}(0) ; \Delta_{\pi}(\sigma)$ строится аналогично.

Функция $\tilde{f}^{\prime} \in C_{[0, \pi]}^{2}$ удовлетворяет краевым условиям (3.1) и вследствие теоремы Стеклова $[7,11]$ разложима в регулярно сходящийся ряд Фурье по собственным функциям (3.2) задачи Штурма-Лиувилля (3.1):

$$
\tilde{f}^{\prime}(\sigma)=\sum_{n=1}^{N} \phi_{n} w_{n}(\sigma)+\delta_{f^{\prime}}(\sigma), \quad\left\|\delta_{f^{\prime}}\right\|_{C}<\frac{\varepsilon}{2 \pi}, \quad \phi_{n}=\frac{\left(\tilde{f}^{\prime}, w_{n}\right)}{\left(w_{n}, w_{n}\right)}
$$

где $(f, g)=\int_{0}^{\pi} f \bar{g} d \sigma$. В силу равномерной сходимости этот ряд можно почленно интегрировать. С учетом (3.2) получим

$$
\tilde{f}(\sigma)=\sum_{n=0}^{N} \tilde{f}_{n} u_{n}(\sigma)+\delta_{f}(\sigma), \quad\left\|\delta_{f}\right\|_{C}<\frac{\varepsilon}{2}, \quad \tilde{f}_{n}=-\frac{\phi_{n}}{\omega_{n}} .
$$

Постоянная интегрирования равна $\tilde{f}_{0}$. Из неравенств в (3.3) и (3.5) следует завершающая доказательство оценка:

$$
\left\|f(\sigma)-\sum_{n=0}^{N} \tilde{f}_{n} u_{n}(\sigma)\right\|_{C}<\varepsilon .
$$

К гладкой функции $\tilde{f}(\sigma) \in C_{[0, \pi]}^{3}$, удовлетворяющей краевым условиям (3.3), ее ряд Фурье (3.5) сходится в норме $C$, т.е. равномерно, и коэффициенты этого ряда $\tilde{f}_{n}$ можно находить как с помощью скалярного произведения (1.13) по типу (1.20), так и посредством предварительного определения $\phi_{n}$. Эти способы эквивалентны, так как $\left(f^{\prime}, w_{n}\right)=-\omega_{n}\left\langle f, u_{n}\right\rangle$ и $\left(w_{n}, w_{n}\right)=\left\langle u_{n}, u_{n}\right\rangle$.

В случае $f \in C_{[0, \pi]}$ для равномерной сходимости ее ряда Фурье требуется выполнение дополнительных условий гладкости [12]. Естественно предполагать, что эти условия выполнены для определяюших движение струны функций (1.19) и их первых и вторых производных, что обеспечивает сходимость и дифференцируемость соответствующих рядов на $(0, \pi)$. 
Отметим, что система, объединяющая $u_{n}$ и $w_{n}$ (равно как и система их линейных комбинаций $\left.e^{-i \omega_{n} \tau}, n \in \mathbb{Z}\right)$, полна в классе $C_{[-\pi, \pi]}$. Отсюда следует полнота этих систем в $C_{I}$, где $I-$ произвольный отрезок с длиной $2 \pi$.

Доказательство этих утверждений мы не приводим ввиду ограниченности места, а также потому, что справедливость их фактически доказана в разделе 2. Действительно, зададим, например, произвольную функцию $U_{1}^{\mu}(\tau), \tau \in[-\pi, \pi]$ (можно для единственного значения $\mu$ ). Определив однозначно с помощью $(2.15)$ на том же отрезке $\Psi_{ \pm}^{\prime \mu}(\tau)$, мы найдем функцию $X^{\mu}(\tau, \sigma)$ - решение задачи (1.3)-(1.9) (с точностью до константы интегрирования $\alpha_{0}^{\mu}$ ) в треугольнике: $\sigma \geq 0, \tau+\sigma \leq-\pi, \tau-\sigma \geq-\pi$. Это позволяет определить функции (1.19), коэффищиенты ряда $\alpha_{n}^{\mu}(1.20)$ и с помощью $(2.19)$ - коэффициенты $\beta_{n}^{\mu}$ разложения в ряд (2.17) заданной функции $U_{1}^{\mu}(\tau)$.

Сходяшийся ряд (1.18) порождает физическое движение струны только в том случае, если выполнены условия ортонормальной калибровки в виде $(1.2)$ или $(2.2)$, а также требование (натуральности параметра) (1.8) или (2.13). Им должны удовлетворять ряд (1.18) и порождаемые им ряды (2.18). Можно также воспользоваться рядами (2.17), которые равенствами (2.19) взаимо однозначно связаны с (1.18). В силу соотношений $(2.6),(2.11)-(2.13)$ условия $(2.2)$ и (1.8) эквивалентны условиям $U_{a}^{2}(\tau)=1$ и (2.14). Подставим в эти условия ряды (2.17), считая их абсолютно сходящимися:

$$
\begin{aligned}
\sum_{m, n=-\infty}^{\infty} R_{a}^{m n}\left(\beta_{m} \beta_{n}\right) e^{-i\left(\omega_{m}+\omega_{n}\right) \tau} & =1, \\
\sum_{m, n=-\infty}^{\infty} T_{a}^{m n}\left(\beta_{m} \beta_{n}\right) e^{-i\left(\omega_{m}+\omega_{n}\right) \tau} & =Q_{a}^{2} .
\end{aligned}
$$

Здесь $R_{1}^{m n}=1, R_{2}^{m n}=\Theta_{m} \Theta_{n}, T_{a}^{m n}=\omega_{m} \omega_{n} R_{a}^{m n}$. Ниже мы установим, что лишш три из четырех условий (3.6) независимы (как и в системе (1.8)-(2.2)).

Покажем, что равенства (3.6) возможны только в случае

$$
\left(\beta_{m} \beta_{n}\right)=0 \quad \forall m, n \in \mathbb{Z}, \quad m+n \neq 0 .
$$

В силу (2.19) условия $\left(\beta_{m} \beta_{n}\right) \equiv \eta_{\mu \nu} \beta_{m}^{\mu} \beta_{n}^{\nu}=0$ эквивалентны $\left(\alpha_{m} \alpha_{n}\right)=0$.

Предварительно исследуем свойства сумм $\omega_{m}+\omega_{n}$ корней уравнения (1.16).

Лемма. Расстояния $\Delta_{n}=\omega_{n+1}-\omega_{n}$ между соседними неотрииательными корнями уравнения (1.16) $\left(Q_{a}>0\right)$ строго монотонно возрастают с ростом $n$ :

$$
\Delta_{n}>\Delta_{n-1} \quad \forall n \geq 1 .
$$

ДокАЗАТЕльСтво. Преобразуем (1.16) к виду

$$
\left(Q_{1}+Q_{2}\right)^{-1}\left(\omega-Q_{1} Q_{2} / \omega\right)=\operatorname{ctg} \pi \omega,
$$

пополнив при этом множество корней (3.8) значением $\omega_{0}=0$. Положительные корни $(3.8) \omega_{n}$ определяются точками пересечения графика функции $F(\omega)=\left(Q_{1}+Q_{2}\right)^{-1} \times$ $\left(\omega-Q_{1} Q_{2} / \omega\right)$ с $n$-й ветвью котангенса, поэтому

$$
n-1<\omega_{n}<n \quad \forall n \geq 1 .
$$


Взяв равенство (3.8) для значений $\omega=\omega_{n}$ и $\omega=\omega_{n+1}$, вычтем первое из второго. Реализовав тождество $\operatorname{ctg} x-\operatorname{ctg} y=(1+\operatorname{ctg} x \cdot \operatorname{ctg} y) / \operatorname{ctg}(y-x)$, получим для $\Delta_{n}=$ $\omega_{n+1}-\omega_{n}$ выражение

$$
\Delta_{n}=\frac{\left(Q_{1}+Q_{2}\right) \omega_{n} \omega_{n+1}}{Q_{1} Q_{2}+\omega_{n} \omega_{n+1}} \cdot \frac{1+\operatorname{ctg} \pi \omega_{n+1} \cdot \operatorname{ctg} \pi \omega_{n}}{-\operatorname{ctg} \pi \Delta_{n}} .
$$

Рассмотрим функцию $\Phi(\Delta) \stackrel{\text { def }}{=} \Delta^{2}-\left(Q_{1}+Q_{2}\right) \Delta \operatorname{ctg} \pi \Delta$, строго монотонно возрастаюшую на интервале $(0,1)$. Так как $\Delta_{n}>0$, а неравенство $\Delta_{n}<1$ следует из строгой монотонности $F(\omega)$ и свойств котангенса, то для доказательства леммы достаточно доказать, что $\forall n \geq 1: \Phi\left(\Delta_{n}\right)>\Phi\left(\Delta_{n-1}\right)$.

С помощью (3.8) преобразуем (3.10) к виду (здесь $\left.\Omega_{n}=Q_{1} Q_{2}+\omega_{n} \omega_{n+1}\right)$

$$
\Phi\left(\Delta_{n}\right)=\Delta_{n}^{2}+\Omega_{n}+\left[\left(Q_{1}^{2}+Q_{2}^{2}\right) \omega_{n} \omega_{n+1}-Q_{1} Q_{2}\left(\omega_{n}^{2}+\omega_{n+1}^{2}\right)\right] / \Omega_{n}
$$

Отметим, что данное выражение справедливо и для $n=0$, так как из (3.8) следует $\Phi\left(\Delta_{0}\right)=\Phi\left(\omega_{1}\right)=Q_{1} Q_{2}$.

Вычитая $\Phi\left(\Delta_{n-1}\right)$ из $\Phi\left(\Delta_{n}\right)$, получим после преобразований

$\Phi\left(\Delta_{n}\right)-\Phi\left(\Delta_{n-1}\right)=\left(\omega_{n+1}-\omega_{n-1}\right) \omega_{n}\left[Q_{1} Q_{2}\left(Q_{1}^{2}+Q_{2}^{2}-Q_{1} Q_{2}+G_{n}\right)+H_{n}\right] \Omega_{n}^{-1} \Omega_{n-1}^{-1}$,

где $G_{n}=\left(\omega_{n+1}-\omega_{n}\right)\left(\omega_{n+1}+\omega_{n-1}\right)+\omega_{n}^{2}+\omega_{n-1}^{2}, H_{n}=\omega_{n+1} \omega_{n} \omega_{n-1}\left(\omega_{n+1}-\omega_{n}+\right.$ $\left.\omega_{n-1}\right)$. Для всех $n \geq 1$ очевидна положительность этой разности, так как по определению $\omega_{n}>\omega_{n-1} \geq 0$, кроме того $Q_{1}^{2}+Q_{2}^{2} \geq 2 Q_{1} Q_{2}$.

Перейдем к доказательству утверждения (3.7) и следующей теоремы.

Теорема 2. Регулярно сходящиеся ряды (2.17) удовлетворяют условиям (3.6) только при выполнении равенств (3.7). Эти ряды представляют собой конечные суммы, содержсашие не более $D$ слагаемых $\left(D-\right.$ размерность $\left.\mathbb{R}^{1, D-1}\right)$.

ДокАЗАТЕЛЬСтво. Ряды (3.6) сходятся регулярно, если слагаемые перенумерованы в порядке возрастания $\omega_{m}+\omega_{n}$ (обозначим эти величины $\chi_{l}$ ). Перенеся постоянные в левую часть, каждое из уравнений (3.6) представим в виде $\sum_{l} \zeta_{l} e^{-i \chi_{l} \tau}=0$. Проведя преобразование Фурье обеих частей этого равенства (ряд допускает почленное интегрирование), мы убедимся, что оно возможно только при $\zeta_{l}=0(\forall l)$.

Величина $\zeta_{l}$ равна сумме всех слагаемых вида $R_{a}^{m n}\left(\beta_{m} \beta_{n}\right)$ (или $T_{a}^{m n}\left(\beta_{m} \beta_{n}\right)$ ), отвечающих одной и той же сумме частот $\chi_{l}=\omega_{m}+\omega_{n}$. Если данное $\chi_{l}$ отвечает единственной паре $m$ и $n$, то для этой пары из $\zeta_{l}=0$ следует равенство (3.7). Исследуем все случаи совпадения сумм двух различных частот.

Покажем, что связывающее корни уравнения (1.16) равенство

$$
\omega_{m}+\omega_{n}=\omega_{i}+\omega_{j}, \quad n \neq i, \quad n \neq j,
$$

невозможно, если все частоты, входяшие в (3.11), неотрицательны. Полагая без ограничения обшности, что $m \geq n, i \geq j, n>j$, и обозначив $l=m-j, k=i-n$ (очевидно, что $l, k \geq 1)$, перепишем (3.11) в виде

$$
\omega_{j+l}-\omega_{j}=\omega_{n+k}-\omega_{n} \Leftrightarrow \Delta_{j}+\cdots+\Delta_{j+l-1}=\Delta_{n}+\cdots+\Delta_{n+k-1} .
$$


Из леммы следует, что при $j<n$ имеют место неравенства $\Delta_{j}<\Delta_{n}, \Delta_{j+1}<$ $\Delta_{n+1}, \ldots$, поэтому при $l=k$ равенство (3.12) невозможно. Предположим, что $l \geq k+1$. Оценив левую часть (3.12) на основании (3.9): $\omega_{j+l}-\omega_{j}>j+l-1-j=l-1 \geq k$, и заметив, что правая часть меншше $k$, так как все $\Delta_{q}<1$, мы получаем противоречие.

Из изложенного следует, что равенство (3.11) возможно только в случае, если три из входяших в (3.11) частот положительны, а одна отрицательна (или наоборот). При этом коэффициент $\zeta_{l}$ при слагаемом ряда (3.6) с $e^{-i \chi_{l} \tau}$, где $\chi_{l}=\omega_{m}+\omega_{n}=\omega_{i}-\omega_{j}$, $m, n, i, j>0$, будет содержать (вследствие доказанного выше) не более двух различных слагаемых, а равенства $\zeta_{l}=0$ для $U_{1}^{\mu}$ будут иметь вид (при $\left.m \neq n\right)$

$$
2\left(\beta_{m} \beta_{n}\right)+2\left(\beta_{i} \beta_{-j}\right)=0, \quad 2 \omega_{m} \omega_{n}\left(\beta_{m} \beta_{n}\right)-\omega_{i} \omega_{j} 2\left(\beta_{i} \beta_{-j}\right)=0 .
$$

Мы учли, что $\omega_{-j}=-\omega_{j}$. Эти уравнения совместны только при $\left(\beta_{m} \beta_{n}\right)=\left(\beta_{i} \beta_{-j}\right)=0$ (то же и в случае $m=n$ ), что доказывает утверждение (3.7).

Покажем, что условия (3.6) выполняются только в том случае, когда вешественный вектор $\beta_{0}^{\mu}$ отличен от нуля и времениподобен $\left(\beta_{0}^{2}>0\right)$.

Разложим $\beta_{n}^{\mu}, \quad n \neq 0$, на вешественную и мнимую части: $\beta_{n}^{\mu}=\varrho_{n}^{\mu}+i \vartheta_{n}^{\mu}$. Условие (3.7) $\left(\beta_{n} \beta_{n}\right)=0$ приводит к соотношениям: $\varrho_{n}^{2}=\vartheta_{n}^{2},\left(\varrho_{n} \vartheta_{n}\right)=0$. Два ортогональных вешественных вектора в пространстве Минковского $\mathbb{R}^{1, D-1}$ не могут быть времениподобными. Следовательно, слагаемые в (3.6) с $m=-n$ (в силу (3.7) остальные не учитываем) при $n \neq 0$ отрицательны или равны нулю, так как $\left(\beta_{n} \beta_{-n}\right)=\varrho_{n}^{2}+\vartheta_{n}^{2} \leqslant 0$. Введя обозначение $b_{n}=2 \sqrt{-\varrho_{n}^{2}}, b_{n} \geq 0$, представим первое из уравнений (3.6) в виде $U_{1}^{2}=\beta_{0}^{2}-\sum_{n=1}^{\infty} b_{n}^{2}=1$. Отсюда следует времениподобность $\beta_{0}^{\mu}$.

Из соотношений $(3.7)\left(\beta_{n} \beta_{0}\right)=0(\forall n \neq 0)$ вытекает, что все $\varrho_{n}^{\mu}$ и $\vartheta_{n}^{\mu}$ ортогональны $\beta_{0}^{\mu}$. Следовательно, ненулевые $\varrho_{n}^{\mu}$ и $\vartheta_{n}^{\mu}-$ пространственноподобные векторы, и справедливо представление

$$
2 \beta_{n}^{\mu}=b_{n}\left(e_{n}^{\mu}+i g_{n}^{\mu}\right), \quad e_{n}^{2}=g_{n}^{2}=-1, \quad\left(e_{n} g_{n}\right)=0, \quad n \geq 1 .
$$

Из (3.7) следует, что $\beta_{0}^{\mu}$ и все $e_{n}^{\mu}$ и $g_{n}^{\mu}(n \geq 1)$ взаимно ортогональны.

Осталось доказать, что всякое семейство векторов $\left\{\beta_{n}^{\mu}\right\}$ в $\mathbb{R}^{1, D-1}$, включающее $\beta_{0}^{\mu}$ $\left(\beta_{0}^{2}>0\right)$ и $\overline{\beta_{n}^{\mu}}$ наряду с $\beta_{n}^{\mu}$, удовлетворяющее условиям (3.7), содержит не более $D$ ненулевых векторов. Предположив противное, мы построим, введя вместо $\beta_{n}^{\mu}, \overline{\beta_{n}^{\mu}}$ их линейные комбинации $e_{n}^{\mu}, g_{n}^{\mu}$, ортогональную систему из более чем $D$ неизотропных векторов. Такая система будет линейно независимой, что невозможно в $\mathbb{R}^{1, D-1}$ (линейном пространстве размерности $D)$.

СЛЕДСТВИЯ И ЗАМЕЧАнИЯ. 1. Число различных частот $\omega_{n}>0$ в рядах $(2.17)$ не превосходит целой части $(D-1) / 2$ (обозначим $\left.N_{D}=[(D-1) / 2]\right)$.

2. Вследствие соотношений (2.18) вывод теоремы о конечности числа слагаемых полностью относится и к ряду (1.18), который для движений струны, удовлетворяюших условиям (1.2) и (1.8), должен иметь вид

$$
X^{\mu}(\tau, \sigma)=\alpha_{0}^{\mu}+p_{0}^{\mu} \tau+C_{1} \sum_{j=1}^{N_{D}} \frac{b_{n_{j}}}{\omega_{n_{j}}} u_{n_{j}}(\sigma)\left[e_{n_{j}}^{\mu} \sin \omega_{n_{j}} \tau-g_{n_{j}}^{\mu} \cos \omega_{n_{j}} \tau\right]
$$

векторы (3.13) $e_{n_{j}}^{\mu}, g_{n_{j}}^{\mu}$ и $p_{0}^{\mu}$ взаимно ортогональны, $\omega_{n_{j}}>0-$ корни (1.16). 
3. Константы $p_{0}^{\mu}=C_{1} \beta_{0}^{\mu}$ и $b_{n_{j}}$ должны удовлетворять четырем условиям (3.6), из которых в качестве трех независимых можно взять

$$
\beta_{0}^{2}-\sum_{j=1}^{N_{D}} b_{n_{j}}^{2}=1, \quad \sum_{j=1}^{N_{D}} \omega_{n_{j}}^{2} b_{n_{j}}^{2}=Q_{1}^{2}, \quad \sum_{j=1}^{N_{D}} \omega_{n_{j}}^{2} \frac{\omega_{n_{j}}^{2}+Q_{1}^{2}}{\omega_{n_{j}}^{2}+Q_{2}^{2}} b_{n_{j}}^{2}=\frac{k_{1}^{2}}{k_{2}^{2}} Q_{2}^{2} .
$$

Четвертое условие - линейная комбинация этих трех с коэффициентами соответственно $Q_{1}^{2},(-1)$ и 1.

4. Доказанные лемма и теоремы (следовательно, и выражение (3.14)) справедливы и в случаях, когда на одном из концов $m_{a}=0$ или $m_{a} \rightarrow \infty$, а частоты $\omega_{n}$ определяются уравнениями (2.21) и (2.22). При этом сохраняются лишш два из условий (3.15), относящихся к другому концу. Из коэффициентов $c_{n}$ в ряду (2.23) отличен от нуля лишь $c_{0}$.

5. В пространствах Минковского $\mathbb{R}^{1,3}(D=4)$ и $\mathbb{R}^{1,2}$ число $N_{D}=1$, т.е. возможно движение изучаемого класса только с одной частотой $\omega_{n}$ :

$$
X^{\mu}(\tau, \sigma)=\alpha_{0}^{\mu}+p_{0}^{\mu} \tau+B_{n} u_{n}(\sigma)\left[e_{n}^{\mu} \sin \omega_{n} \tau-g_{n}^{\mu} \cos \omega_{n} \tau\right]
$$

где $B_{n}=C_{1} Q_{1} / \omega_{n}^{2}, p_{0}^{2}=C_{1}^{2}\left(1+Q_{1}^{2} / \omega_{n}^{2}\right)$ определяются условиями (3.15). Всякую мировую поверхность (3.16) с точностью до преобразования Пуанкаре в $\mathbb{R}^{1, D-1}$ задают следующие произвольные параметры: $Q_{1}, Q_{2}$ (частота $\omega_{n}$ определяется ими с точностью до $n$ ) и масштабный фактор $C_{1}$ (или $k_{1}$ ).

Лишь одно движение струны вида (3.16), а именно движение с минимальной частотой $\omega_{1}>0$, не имеет на мировой поверхности особенностей вида $\dot{X}^{2}=0$. Определенная с помощью монотонной функции $u_{1}(\sigma)(1.15)$ мировая поверхность этой струны $(3.16)$ представляет собой хорошо известный геликоид, заметаемый равномерно врашаюшейся струной, которая сохраняет форму отрезка прямой $[1,3,5,13]$. При этом в системе координат (связанной с $p_{0}^{\mu}$ ) неподвижна точка, соответствующая единственному нулю функции $u_{1}(\sigma)$.

Мировые поверхности вида (3.16) с частотами $\omega_{n}, n \geq 2$, будут иметь особенности - при $\sigma \in[0, \pi]$ сушествуют $(n-1)$ линий $\sigma=$ const (нули функции $u_{n}^{\prime}(\sigma)$ ), на которых $X^{\prime \mu}$ обращается в нуль, что влечет обращение в нуль индуцированной метрики $\left(\dot{X}^{2}=X^{\prime 2}=0\right)$. Соответствующие точки движутся со скоростью света. Физически это можно воспринимать как врашаюшуюся струну, сложенную $n$ раз, или как $n$ совмешенных в пространстве струн, участвуюших в едином врашении. Концы струн, отвечаюшие $X^{\prime \mu}=0$, безмассовы, первая из струн имеет массы на концах $m_{1}$ и 0 , последняя -0 и $m_{2}$.

Интересно отметить, что этих особенностей $\left(\dot{X}^{2}=0\right)$ лишены движения струны в пространствах размерности $D \geq 5$, определяемые выражением (3.14) и содержашие $N_{D}=2$ и более различных частот $\omega_{n}$, например движение, определяемое вектором $U_{1}^{\mu}(\tau)=\beta_{0}^{\mu}+b_{1}\left(e_{1}^{\mu} \cos \omega_{1} \tau+g_{1}^{\mu} \sin \omega_{1} \tau\right)+b_{2}\left(e_{2}^{\mu} \cos \omega_{2} \tau+g_{2}^{\mu} \sin \omega_{2} \tau\right)$. Множество этих мировых поверхностей описывается с точностью до движения в $\mathbb{R}^{1, D-1}$ удовлетворяюшими условиям (3.15) параметрами $b_{n_{j}}, \beta_{0}^{2}, C_{a}$ и $Q_{a}-$ всего $\left(N_{D}+2\right)$ независимыми параметрами.

\section{ЗАКЛЮЧЕНИЕ}

Итак, нами классифицированы движения релятивистской струны (1.1) (включая случаи $m_{a}=0$ и $m_{a} \rightarrow \infty$ на одном из коншов), допускаюшие при вьполнении условий (1.2) 
линеаризацию краевых условий вследствие ограничения (1.8) на траекториях каждого из концов струны с ненулевой конечной массой. В $3+1$-мерном пространстве Минковского все эти движения являются плоскими, периодическими, имеют вид (3.16) и сводятся к равномерному врашению прямолинейной струны или нескольких таких струн. Это подтверждает результат работы [13], в которой единственность геликоидального решения данной задачи была найдена в калибровке $\tau=t$. Таким образом, использование линейности задачи в целях квантования приводит к квантованию единственного движения.

В пространствах $\mathbb{R}^{1, D-1}, D \geq 5$, возможны более сложные непериодические движения (3.14) рассматриваемого класса с $N_{D}=[(D-1) / 2]$ несоизмеримыми частотами.

\section{Список литературы}

[1] B.M. Barbashov, V.V. Nesterenko. Introduction to the relativistic string theory. Singapore: World Scientific, 1990; Б.М. Барбашов, В.В. Нестеренко. Модель релятивистской струны в физике адронов. М.: Энергоатомиздат, 1987.

[2] М. Грин, Дж. Швари, Э. Виттен. Теория суперструн. Т. 1, 2. М.: Мир, 1990.

[3] A. Chodos, C.B. Thom. Nucl. Phys. 1974. V. B72. № 3. P. 509-522.

[4] B.M. Barbashov. Nucl. Phys. 1977. V. B129. № 1. P. 175-188.

[5] Б.М. Барбашов, А.М. Червяков. ТМФ. 1988. Т. 74. № 3. С. 430-439; 1991. Т. 89. № 1. C. $105-120$.

[6] Б.М. Барбашов, В.В. Нестеренко. ТМФ. 1977. Т. 31. № 3. С. 291-299.

[7] В.С. Владимиров. Уравнения математической физики. М.: Наука, 1980.

[8] Н.С. Кошляков, Э.Б. Глинэр, М.М. Смирнов. Уравнения в частных производных математической физики. М.: Высшая школа, 1970.

[9] Б.М. Барбашов, Г.С. Шаров. ТМФ. 1994. Т. 101. № 2. С. 253-271.

[10] Г.С. Шаров. ТМФ. 1995. Т. 102. № 1. С. 150-159.

[11] В.А. Стеклов. Основные задачи математической физики. М.: Наука, 1983.

[12] A.Н. Колмогоров, С.В. Фомин. Элементы теории функций и функционального анализа. М.: Наука, 1981.

[13] B.M. Barbashov. Classical dynamics of rotating string with massive ends. In: Strong Interactions at Long Distances. Palm Harbor, FL USA: Hadronic Press, 1995. P. 257.

Поступила в редакцию 29.XI.1995 г., после доработки

13.V.1996 г.

\section{V.P. Petrov, G.S. Sharov}

\section{CLASSIFICATION OF MOTIONS OF A RELATIVISTIC STRING WITH MASSIVE ENDS WITH LINEARIZABLE BOUNDARY CONDITIONS}

We classified all motions (world surfaces) of a relativistic string with massive ends, for which equations of motion and boundary conditions can be linearized through a natural parametrization of the end's trajectories. These motions can be represented as Fourier series with eigenfunctions of some generalization of the Sturm-Liouville problem. Completeness of a set of these eigenfunctions in class $C$ is proved. It is shown that in $2+1$ and $3+1$-dimensional Minkowski spaces all these motions reduce to an uniform rotation of a straight string or some such spatially coincident strings (world surface is helicoid). In spaces with higher dimensionality other non-trivial motions of the investigated type are possible. 